\title{
A Cluster Allocation and Routing ALGORITHM BASED ON NODE DENSITY FOR EXTENDING THE LIFETIME OF WIRELESS SENSOR NETWORKS
}

\author{
Wernhuar Tarng, Hao-Wei Lin, and Kuo-Liang Ou \\ Graduate Institute of Computer Science, National Hsinchu University of Education, \\ Hsinchu, Taiwan \\ wtarng@mail.nhcue.edu.tw, hil15201908@gmail.com, klou@mail.nhcue.edu.tw
}

\begin{abstract}
The electricity of sensor nodes in wireless sensor networks is very limited, so it is an important research topic to deploy the sensor nodes and cooperate with an efficient routing algorithm for extending the network lifetime. In the related research, LEACH routing algorithm randomly selects cluster heads in each round to form a cluster network, which may cause additional power consumption and inability to maintain the optimal routes for data transmission. The cluster allocation and routing algorithm proposed in this study is based on the cluster architecture of LEACH, and the objective is to produce clusters with more sensor nodes to balance energy consumption of cluster head. For indirect-transmission routing algorithms, the sensor nodes near the base station may consume more energy due to a larger amount of data transmission. Therefore, this study proposed to increase the node density near the base station during deployment to compensate for the requirement of high energy consumption. The experimental results show that the proposed algorithm based on node density distribution can efficiently increase the lifetime of wireless sensor networks.
\end{abstract}

\section{KEYWORDS}

Wireless Sensor Networks, Routing Algorithms, Cluster Networks, Node Density, Network Lifetime

\section{INTRODUCTION}

The wireless sensor network (WSN) was initially a research project directed by UC Berkeley, where they used the micro-electro-mechanical technology to design wireless sensors about the size of a coin, also called smart dusts. A WSN consists of spatially distributed autonomous sensors to monitor physical or environmental conditions, such as temperature, sound, vibration, pressure, pollutants and motion to cooperatively pass their data through the network to the base station [1]. The WSN was first used to collect data in the battle fields. Due to its small size, low price and various functions, the WSN has now been used in daily applications, for example, taking care of elder people, vibration detection of bridge piers, detection of fire and $\mathrm{CO}_{2}$ emission, and so on.

In the data transmission of WSN, the strength of signals is highly related to the distance, i.e., the longer the distance, the weaker the signal and the more energy consumed. When the distance is too long, it may cause the sensor nodes to exhaust energy quickly and thus affect the network lifetime. As a result, how to fully utilize the electric power of sensor nodes for continuous data transmission is an important topic of WSN research. Basically, the ways of data transmission for the WSN can be categorized as direct transmission and indirect transmission [2]. 
International Journal of Computer Science \& Information Technology (IJCSIT) Vol 4, No 1, Feb 2012

\section{A. Direct Transmission}

Each sensor nodes transmit the collected data to the base station directly. In this approach, the data rate is higher since there is no forwarding during transmission. Although the implementation for direction transmission is easier, the data may not be sent to the destination when the application area is very large, or the sensor nodes may die quickly due to high energy consumption. Therefore, this approach is suitable only for small-area applications.

\section{B. Indirect Transmission}

The sensor nodes send the collected data to the neighbour nodes, which will forward the data to the base station. This approach solves the problem of high energy consumption in long-distance transmission, but it may cause some nodes to consume more energy by forwarding data for the other nodes and thus affecting the network lifetime.

Usually, the deployment of a WSN is to scatter a large amount of sensor nodes over the application area randomly. Then, the sensor nodes start to collect and transmit data back to the base station via multi-hop forwarding. Since each node does not know the exact position of other nodes at the beginning, a self-organization protocol [3] is required to connect the sensor nodes to form a communication network for data transmission.

The main objective of a routing algorithm is to find a better way for data transmission to save electric power. Therefore, how to design an efficient routing algorithm to extend the lifetime of WSN has become a very important research topic $[4,5]$. LEACH is a classic routing protocol [6] for cluster WSN because it can balance energy consumption within a cluster, and hence extend the network lifetime. Its operation contains two stages: initial stage and stable stage. In the initial stage, the base station selects a number of nodes as cluster heads based on random thresholds. All other nodes join their nearby clusters by sending out signals and discover the closest cluster heads. When clusters are formed, the wireless sensor network goes into a stable stage. Each node starts to collect and transmit data to its cluster head, and the cluster head forward these data to the base station together with their own collected data.

The reason for LEACH to randomly select a fixed number of cluster heads in each round is to solve the problem of high energy consumption in direct-transmission approach and to balance the power consumption among all sensor nodes. During the operation of each round, LEACH generates a threshold value, which is sent to each sensor node to determine if it can become a cluster head by a probability function. The new cluster head has to aggregate data from sensor nodes in its cluster and transmit the data to the base station. In this way, all sensor nodes take turns to serve as a cluster head to balance the power consumption. Many routing algorithms were developed based on the same approach of LEACH in creating clusters, e.g., PAGASIS [7] and TEEM [8].

PEGASIS works by connecting nodes in series, starting from the farthest node to the base station, to form a linked structure with its neighbour nodes. When all nodes are connected, the head of the linked structure will be selected. Then, every node combines both received data and its own data and sends them towards the head. When all data arrive at the head, it will be forwarded to the base station. Since the route is computed by the greedy algorithm, it is the shortest and consumes less energy than LEACH. However, the greedy algorithm is complicated and thus requires more energy in computation.

TEEN is also based on LEACH to transmit data to the base station periodically. It sets two threshold values, i.e., hard threshold and soft threshold, to avoid the transmission of duplicated data. This approach can save some energy by reducing the amount of data, but it is not suitable for the applications requiring periodical data since the threshold values may not be met in occasion. Therefore, a revised version of TEEN was proposed [9] to remedy the drawback of 
reporting data periodically by reacting to sudden events in real time. Since both algorithms are based on LEACH, the amount of energy saved is still limited.

The coverage ratio of a WSN represents the percentage of area covered by the working sensor nodes among all application area, and it can also be used as an index for data integrity [10]. A WSN can not achieve its function when the coverage ratio is too low. Besides, sensor nodes have more feasible routes for selection to reduce energy consumption when the coverage ratio is high. On the other hand, there often exist some holes [11] to block the shorter routes when the coverage ratio is low, so the sensor nodes may have to use a longer route to bypass the holes for data transmission. As the result, a lower coverage ratio may cause more energy consumption and lower data integrity.

To extend the lifetime of WSN, a cluster allocation and routing algorithm based on node density is proposed in this study. The objective is to solve the problem of LEACH and the related routing algorithms for which the electric power of sensor nodes near the base station may exhaust quickly and thus reduce the coverage ratio.

This study adopts a fixed cluster allocation mechanism and a deployment method based on the node density for extending network lifetime. At the beginning, the algorithm allocates the sensor nodes to a number of clusters, and the goal is to create clusters containing more sensor nodes to share the energy consumption of their cluster head in forwarding data to the base station.

For indirect transmission mode, the sensor nodes near the base station may exhaust their energy quickly due to forwarding data for other sensor nodes, and it may also disable the data transmission of the outer sensor nodes. Therefore, this study proposed to increase the node density near the base station to compensate for the requirement of higher energy consumption in this area. Without changing the total number of sensor nodes, the node density in the outer area is reduced accordingly. The goal of the density deployment method is to remain a high coverage ratio for extending the lifetime of WSN. Simulation results are provided to show the efficiency of this approach.

\section{RELATED METHODS}

The cluster allocation and routing algorithm proposed in this study is based on LEACH's clustered architecture. The difference is that the cluster allocation is done only once at the beginning and remains fixed for the rest of time. Also, the node density near the base station is increased during the deployment stage to achieve a higher coverage ratio and a longer lifetime as well. The methods of high-energy-first mechanism for determining the cluster heads, finding the forwarding node with the smallest angle [12], data compression [13] and sleep mode [14, 15] were also incorporated in the routing algorithm to further reduce the energy consumption and extend the lifetime of WSN. These methods are described in the following.

\section{A. High-Energy-First Method}

In LEACH, a recently retired cluster head still has a chance to be selected again according to the probability function, which may lead to fast exhaustion of its electric power. Therefore, this study adopts the high-energy-first method to select cluster heads in each round to remedy this drawback. After collecting data, each sensor node has to send out data together with the information about its remaining electric power, and then the base station can decide which nodes are to be selected as cluster heads in the next round using broadcast messages.

\section{B. Determining Forwarding Node}

In each round, a cluster head has to determine the forwarding node, which is also a cluster head in its neighbourhood. The most direct forwarding route is determined based on the angle between the directions of the base station and the forwarding node. The neighbour cluster head 
with the smallest angle is chosen as the candidate first, and the purpose is to use the shortest route to reduce energy consumption in forwarding data. However, if the cluster head with the smallest angle refuses to forward data due to its low electricity, the one with the second smallest angle is chosen next, and so on. If no cluster heads within 90 degree are available, the requesting cluster head must transmit data directly to the base station when the distance is reachable at the cost of consuming more energy; otherwise, it will seek for cluster heads outside 90-degree area as the forwarding node using a longer route.

\section{Data Compression}

In WSN, the amount of data transmitted can also affect the energy consumption of sensor nodes. When a cluster head detects the same or similar data packets which are being transmitted, it can use the data compression method to filter out similar data packets. This method can reduce the amount of data and therefore save some energy.

\section{Sleep Mode}

When sensor nodes are scattered randomly during the deployment of a WSN, some nodes may be too close to each other and collect the same data. Therefore, using sleep mode can reduce the data amount and energy consumption. In this study, a grouping method is used to divide sensor nodes into a number of groups within a cluster. In each group, the node with more electric power is selected as the active node while the other nodes may enter sleep mode. The main objective of the grouping method is to evenly distribute active nodes in each cluster to reduce data similarity. Furthermore, the ratio of active nodes and the group size can be adjusted according to the requirement of data precision.

\section{Cluster Allocation And Routing}

This study adopted a fixed cluster allocation algorithm similar to the initial stage of LEACH. At first, the base station sends a threshold value to all sensor nodes for the selection of cluster heads. Then, this study used the cluster allocation algorithm to create clusters with more sensor nodes. After that, each sensor node sends the information back to the base station for later usage, including node number, cluster number, location and remaining electricity. As soon as the initialization stage is completed, the WSN begins its operation in each round to collect and transmit data by the routing algorithm.

\section{A. Cluster Allocation}

The concept of set operation is used in dividing the sensor nodes in a WSN into a number of clusters. At the beginning, the initial stage of LEACH is used for selecting cluster heads, and then the cluster heads create their own clusters by communicating with the sensor nodes within the sensing area. For simplicity, the created clusters are defined as the allocated sets, while the sensor nodes not invited by any cluster heads are left in the unallocated set. Then, the allocated sets are sorted by the number of their sensor nodes, and those in the intersected sets are reallocated to the set with more sensor nodes. The objective is to produce clusters with more sensor nodes such that they are more powerful in forwarding data for other nodes. After that, the sets with very few nodes will be deleted, and their nodes are put into the unallocated set.

When all sensor nodes in the intersected sets are re-allocated, each sensor node belongs to a unique cluster. If there are still some sensor nodes in the unallocated set, the algorithm repeats the same process as described above until the remaining sensor nodes have been allocated to a certain cluster. The reason for re-allocating the sensor nodes in the intersected set is to produce clusters with more sensor nodes to compensate for the high energy consumption by cluster heads in forwarding data (Figure 1). The steps for cluster allocation are described in the following: 


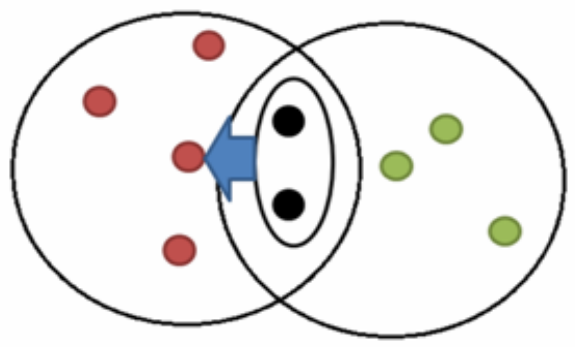

Figure 1. Allocating the intersected set to the set with more nodes

Step1: The initial stage of LEACH is used for selecting cluster heads from all sensor nodes, and then the cluster heads create a number of clusters by communicating with the sensor nodes within the sensing area. The sensor nodes not invited by any cluster heads are assigned in unallocated set $C_{0}$.

Step2: Sort the allocated sets by their node numbers in descending order such that the number of nodes in set $C_{i}$ is greater than or equal to that of set $C_{j}$ if $i<j$.

Step3: Starting from the allocated set with more nodes, assign the nodes in the intersection $C_{i} \cap C_{j}$ to set $C_{i} \forall j \neq i$ and $i<j$. If the assigned cluster head in set $C_{j} \in C_{i} \cap C_{j}$, assign the remaining nodes in $C_{j}$ to $C_{0}$ and remove $C_{j}$ from the list of allocated sets.

Step4: If $C_{0} \neq \phi$, select some nodes in $C_{0}$ as cluster heads to form more clusters. The nodes in the newly created sets are removed from $C_{0}$. Repeat step2 to step4 until $C_{0}=\phi$ or the condition of the initial coverage ratio is satisfied.

\section{B. Routing Algorithm}

The routing algorithm operates by rounds. After sensor node deployment and cluster allocation, the routing algorithm uses high-energy-first method to select sensor nodes with more electric power as the cluster heads, and determines the forwarding nodes for data transmission. After that, all sensor nodes start to collect data, which are aggregated by the cluster heads and forwarded to the base station. Finally, the base station computes the coverage ratio to decide if the operation will continue or not. If the coverage ratio is acceptable, the system goes to the next round to repeat its operation. The cluster head decides its forwarding node by finding the shortest route to reduce energy consumption. However, the neighbouring cluster heads may refuse to forward data due to insufficient electric power. In that case, the cluster head initiates the dynamic routing process to forward data by a feasible route when its forwarding request is declined by other cluster heads.

As shown in Figure 2, cluster head $A$ selects node 1 as its forwarding node initially, but is declined due to low electric power; it then selects the node with the second smallest angle, i.e., node 4, but is declined again. Then, it selects the node with the third smallest angle, i.e., node 2, but still in vain. The process continues until there are no forwarding nodes in the direction of base station for selection. After that, the cluster head try to transmit data to the base station directly if it is within the reachable range. Otherwise, it will turn to request nodes in the direction greater than 90 degree as a last resort. When a cluster head has to request a forwarding node deviating from the direction of the base station, it means most nodes in the preferred direction are in low-power conditions. Instead of giving up at this moment, the cluster head tries it best by using a detoured route to send out data since the goal is to maintain a high receiving ratio by the base station. 


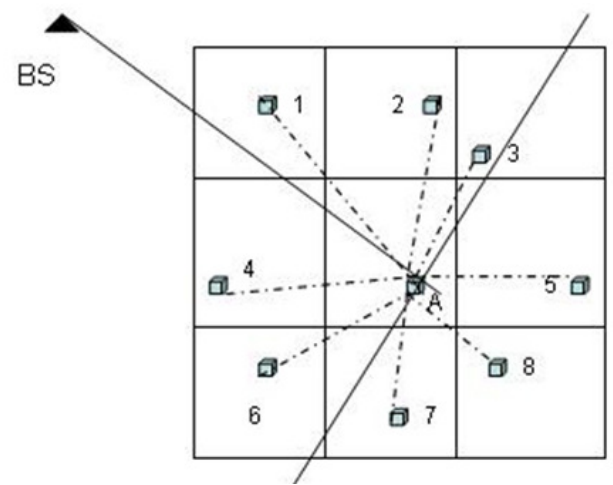

Figure 2. Locating forwarding node in neighbouring clusters

\section{Simulation Results}

This study conducted several simulation experiments to analyze if the proposed algorithm could extend the lifetime of WSN. In addition, this study would like to investigate if raising the node density around the base station without increasing the total number of sensor nodes can deal with the high energy consumption problem in this area. This study also tried to find out a better density proportion for usage in the deployment stage to extend the lifetime of WSN. The results were compared with those of LEACH algorithm under the same conditions.

Before the simulation experiments, this study provides the following analysis about the power consumption by sensor nodes. Basically, the energy required for transmitting a signal is highly related to the distance [16]. The following equation shows the energy consumed when sending a signal to a distance $d$ by an amplifier.

$$
\text { Energy consumption }=\left\{\begin{array}{l}
\varepsilon_{f s} \times d^{2}, \text { if } d \leq d_{0} \\
\varepsilon_{t r} \times d^{4}, \text { if } d>d_{0}
\end{array}\right.
$$

Using $d_{0}$ as a threshold, if the transmission distance is shorter than $d_{0}$, a free-space propagation model is used to calculate the consumed energy, which is proportional to the square of distance. If transmission distance is longer than $d_{0}$, the two-ray ground propagation model is used for calculation and the consumed energy is proportional to the fourth power of distance. In that case, the consumed energy has a great influence on the wireless communication system. In the above equation, $\varepsilon_{f s}$ and $\varepsilon_{t r}$ are the parameters for the free-space propagation model and tworay ground propagation model with their values equal to $10 \mathrm{pJ} / \mathrm{bit} / \mathrm{m}^{2}$ and $0.0013 \mathrm{pJ} / \mathrm{bit} / \mathrm{m}^{4}$, respectively; Here, $d_{0}$ is defined as $\sqrt{\varepsilon_{f_{s}} / \varepsilon_{t r}}$, which is the threshold of transmission distance and its value is about 87.7 . To simplify the computation, it is assumed that $d_{0}=100 \mathrm{~m}$.

For most sensor nodes in WSN, the consumed energy is proportional to the square of distance when collecting and sending data to their cluster head. The cluster allocation algorithm can increase the node density in a cluster and thus reduce the distance and consumed energy in transmitting data. As the operation continues, the sensor nodes near the base station may exhaust their electricity and thus can not forward data for the outer sensor nodes. Consequently, the outer sensor nodes may need to transmit data directly to the base station at a longer distance, so the energy consumed is proportional to the fourth power of distance.

In this study, the simulation programs were written by Java. To obtain more accurate results, all experiments were performed 100 times to compute the average results. The parameters for the experiments are described in the following. The size of the application area is $700 \mathrm{~m} \times 700 \mathrm{~m}$ 
and there are 2000 sensor nodes deployed. The longest transmission distance for a senor node is $200 \mathrm{~m}$. The initial energy in each sensor node equals $2 \mathrm{~J}$, and the energy for sending and receiving data are the same, $50 \mathrm{~nJ} / \mathrm{bit}$. Each data aggregation takes $5 \mathrm{~nJ} / \mathrm{bit}$ with the compression rate $=70 \%$. The amplifier parameters for the two models are $\varepsilon_{f s}=10 \mathrm{pJ} / \mathrm{bit} / \mathrm{m}^{2}$ and $\varepsilon_{t r}=0.0013 \mathrm{pJ} / \mathrm{bit} / \mathrm{m}^{4}$, respectively, and the data rate equals $160 \mathrm{bit} / \mathrm{sec}$.

\section{A. Computing Coverage Ratio}

The coverage ratio of a WSN represents the percentage of area under monitoring (Figure 3), which is computed as the area covered by the working sensor nodes divided by the total application area $\left(700 \mathrm{~m} \times 700 \mathrm{~m}=490000 \mathrm{~m}^{2}\right)$. The sensor nodes can function correctly only when they have enough electricity, so the electric power is the major factor affecting coverage ratio. In this study, the WSN stops its operation if the coverage ratio goes below $80 \%$, and the number of rounds at this time is defined as its lifetime.

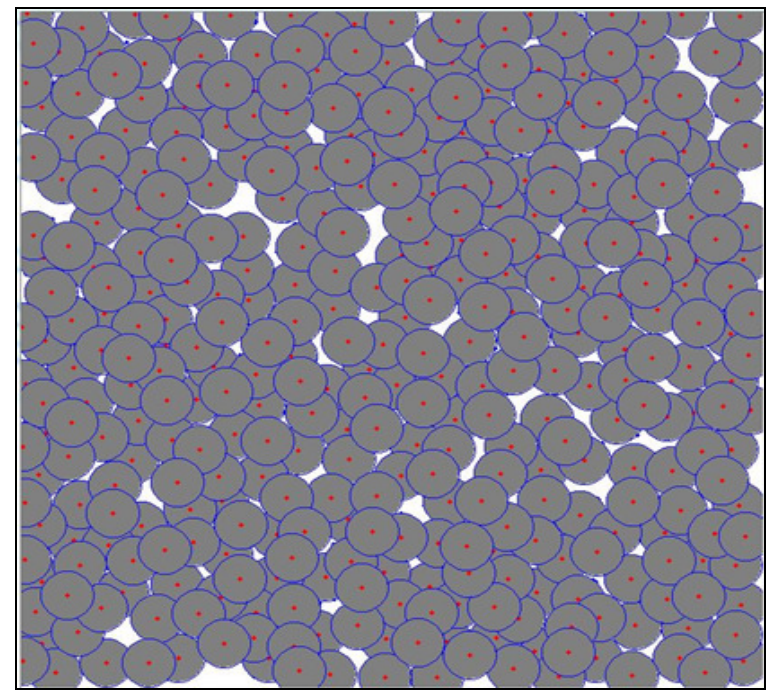

Figure 3. The area covered by working sensor nodes

\section{B. Location of Base Station}

The location of base station has a great influence on the energy consumption of sensor nodes (Table 1). To find out a better location for the base station, an experiment was conducted to simulate the operation of a WSN with the base station situated in three different locations, i.e., upper left (Figure 4), left (Figure 5), and center (Figure 6). In these figures, green spots indicate the base station, and black lines represent the forwarding routes. The node colour is used to represent the status of remaining electric power in a sensor node as follows: blue (sufficient), red (low), and black (exhausted). The algorithm proposed in this study was used for collecting and sending data, and the WSN stops its operation when the coverage ratio goes below $80 \%$. According to the lifetimes of the base station situated at three different locations, it can be seen that the location of the base station plays an important role to affect the lifetime of WSN. The lifetime is the longest when the base station is located at the center. The reason is that there were more sensor nodes to share the high energy consumption around the base station. 
International Journal of Computer Science \& Information Technology (IJCSIT) Vol 4, No 1, Feb 2012

Table 1. Lifetime vs. the location of base station.

\begin{tabular}{|c|c|}
\hline Location of base station & Lifetime (rounds) \\
\hline Upper left & 1500 \\
\hline Left & 2655 \\
\hline Center & 8342 \\
\hline
\end{tabular}

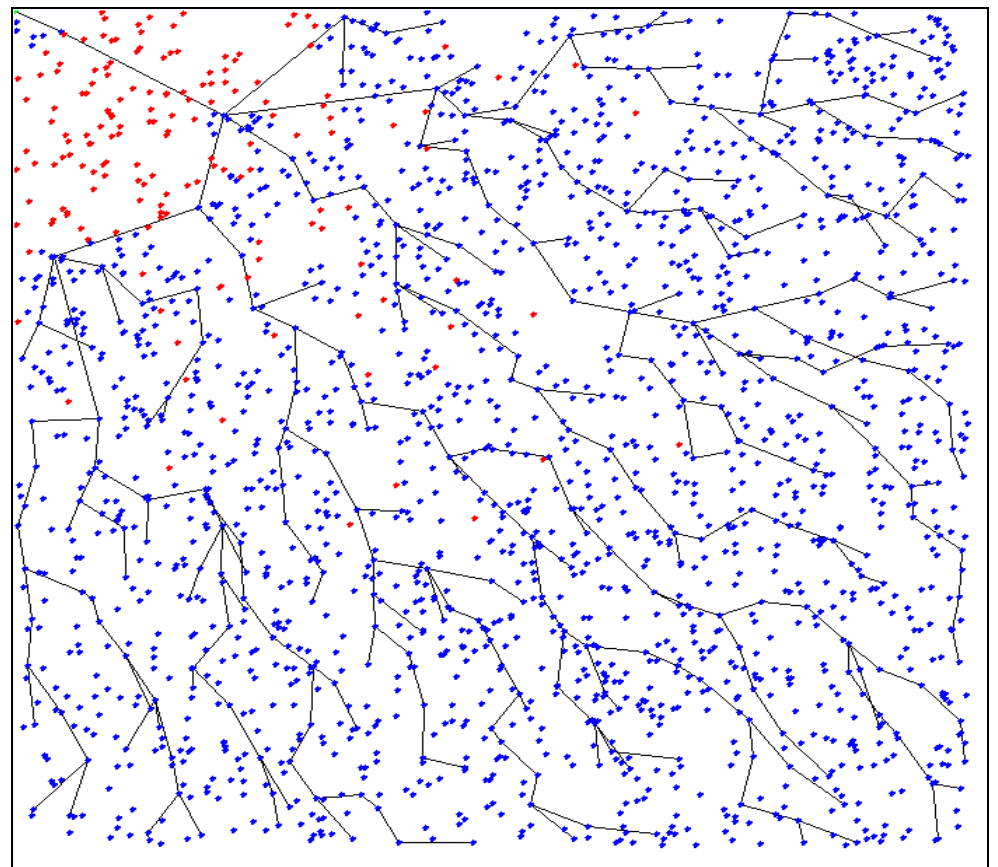

Figure 4. The base station is located at upper left

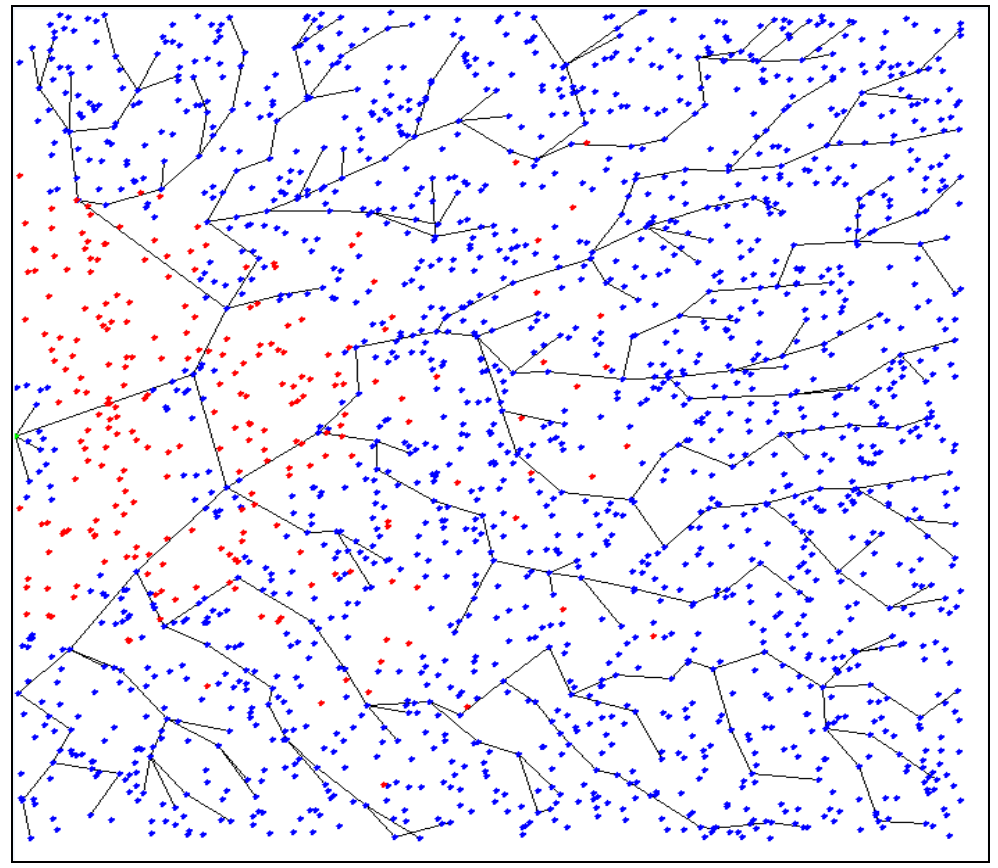

Figure 5. The base station is located at the left 
International Journal of Computer Science \& Information Technology (IJCSIT) Vol 4, No 1, Feb 2012

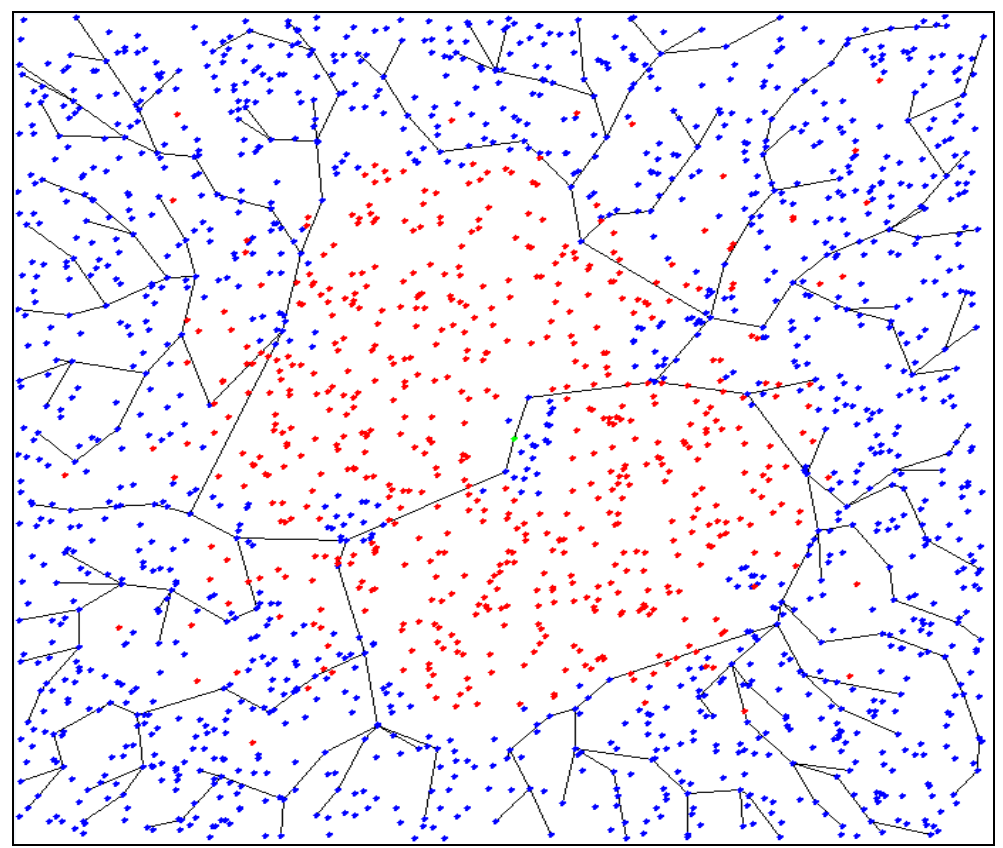

Figure 6. The base station is located at the center

\section{Comparison with LEACH}

In this experiment, 2000 sensor nodes were deployed in a $700 \mathrm{~m} \times 700 \mathrm{~m}$ application area. The proposed algorithm and LEACH were used to collect and transmit data. The lifetimes (in rounds) for both algorithms were recorded until the coverage ratio dropped below $80 \%$. As we can see in Figure 5, the initial coverage ratio is about $99 \%$ for both algorithms. For LEACH, the coverage ratio goes below $80 \%$ at the time of 5002 rounds. For the proposed algorithm, the coverage ratio goes below $80 \%$ at the time of 8342 rounds. Hence, the proposed algorithm can efficiently increase the lifetime of WSN, and the percentage of increment is $66 \%$.

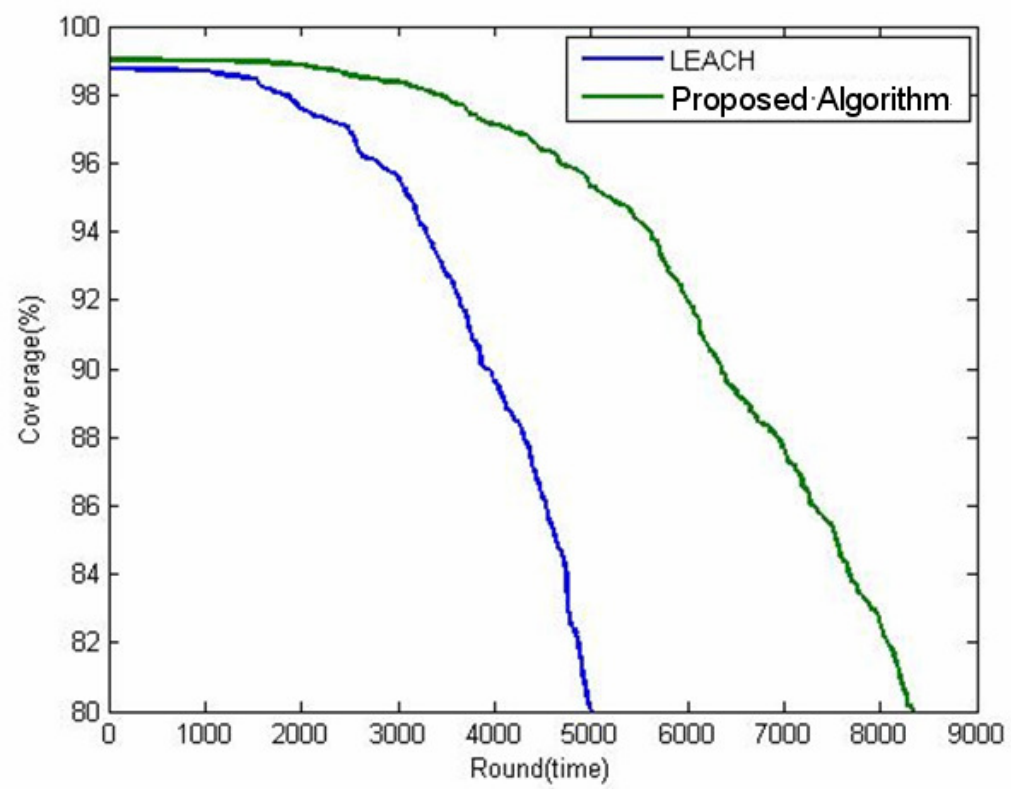

Figure 7. Comparing the lifetimes of LEACH and proposed algorithm. 


\section{Adjusting Node Density}

When the indirect transmission mode is used in WSN, the sensor nodes near the base station often exhaust their energy first due to forwarding data for other nodes, which can also result in the early death of WSN. As we can see from the above results, there are a lot of active sensor nodes in outer areas not able to transmit data to the base station because most of the inner sensor nodes have exhausted their electricity. Hence, this study proposed a density deployment method to raise the density of sensor nodes near the base station without increasing the total number of sensor nodes. The simulation results showed that this approach was very useful in extending the lifetime of WSN.

To find out a better density proportion for deploying sensor nodes, an experiment was conducted by dividing the application environment into 3 areas: (A) the area within $200 \mathrm{~m}$ of the base station, (B) the area of distance between $200 \mathrm{~m}$ and $300 \mathrm{~m}$ from the base station, and (C) the area of distance longer than $300 \mathrm{~m}$ from the base station. According to previous results, the sensor nodes in area A will consume more energy, and those in area B will consume less energy, and those in area $\mathrm{C}$ will consume the least energy.

By adjusting the node density in these 3 areas, this study tried to find out a better density proportion to increase the coverage ratio of WSN and its lifetime as well. There are 2000 sensor nodes deployed according to the designated density in each area. Because the proportion of these 3 areas is $200^{2} \pi:\left(300^{2}-200^{2}\right) \pi:\left(700^{2}-300^{2}\right) \pi=4 \pi: 5 \pi: 49-9 \pi$, if their node densities are the same, the numbers of sensor nodes in these three areas are computed as $\mathrm{N}_{\mathrm{A}}=2000 \times \frac{4 \pi}{49}=513$,

$\mathrm{N}_{\mathrm{B}}=2000 \times \frac{5 \pi}{49}=641$, and $\mathrm{N}_{\mathrm{C}}=2000 \times \frac{(49-9 \pi)}{49}=846$.

The experiment compared the lifetimes (in rounds) for five different density proportions when the coverage ratio dropped below $80 \%$ by the proposed algorithm and LEACH. It is noted that the lifetime for each case was computed by the average of 100 simulation results. The results in Table 2 shows that raising the node density in the area near the base station can increase the lifetime of WSN. When the density proportion is $4: 2.5: 1$, the lifetime for the cluster allocation and routing algorithm is the longest (16789 rounds). The same situation can also be found in LEACH algorithm, and the longest lifetime equals 11379 rounds when the density proportion is $4: 2.5: 1$.

Table 2. Lifetime for different density proportions (the proposed algorithm and LEACH)

\begin{tabular}{|c|c|c|c|c|c|}
\hline \multirow{2}{*}{ Density proportion } & \multicolumn{3}{|c|}{ Node number } & Rounds when coverage ratio dropped below 80\% \\
\cline { 2 - 6 } & A & B & C & Proposed Algorithm & LEACH \\
\hline $1: 1: 1$ & 513 & 641 & 846 & 8342 & 5002 \\
\hline $2: 1.5: 1$ & 724 & 678 & 598 & 13569 & 9932 \\
\hline $3: 2: 1$ & 839 & 699 & 462 & 15073 & 10622 \\
\hline $4: 2.5: 1$ & 912 & 712 & 376 & 16789 & 11379 \\
\hline $5: 3: 1$ & 961 & 721 & 318 & 15598 & 10676 \\
\hline $6: 3.5: 1$ & 998 & 727 & 275 & 16288 & 11019 \\
\hline
\end{tabular}

As we can see from the above results, raising the node density near the base station can increase the lifetime of a WSN. This study further compared the results of LEACH and the proposed algorithm under the density proportion of 4:2.5:1. As shown in Figure 8, the initial coverage ratios for both algorithms are about $95 \%$. The coverage ratio of LEACH drops to $80 \%$ at the time of 11379 rounds, which is increased by $127 \%$ compared to the case without adjusting node density (5002 rounds). The coverage ratio of the proposed algorithm drops to $80 \%$ at the time of 
16789 rounds, increased by $101 \%$ compared to the case without adjusting node density (8342 rounds). For both algorithms, their lifetimes have been increased by more than $100 \%$ (as shown in Table 3) if the proposed density deployment method was applied.

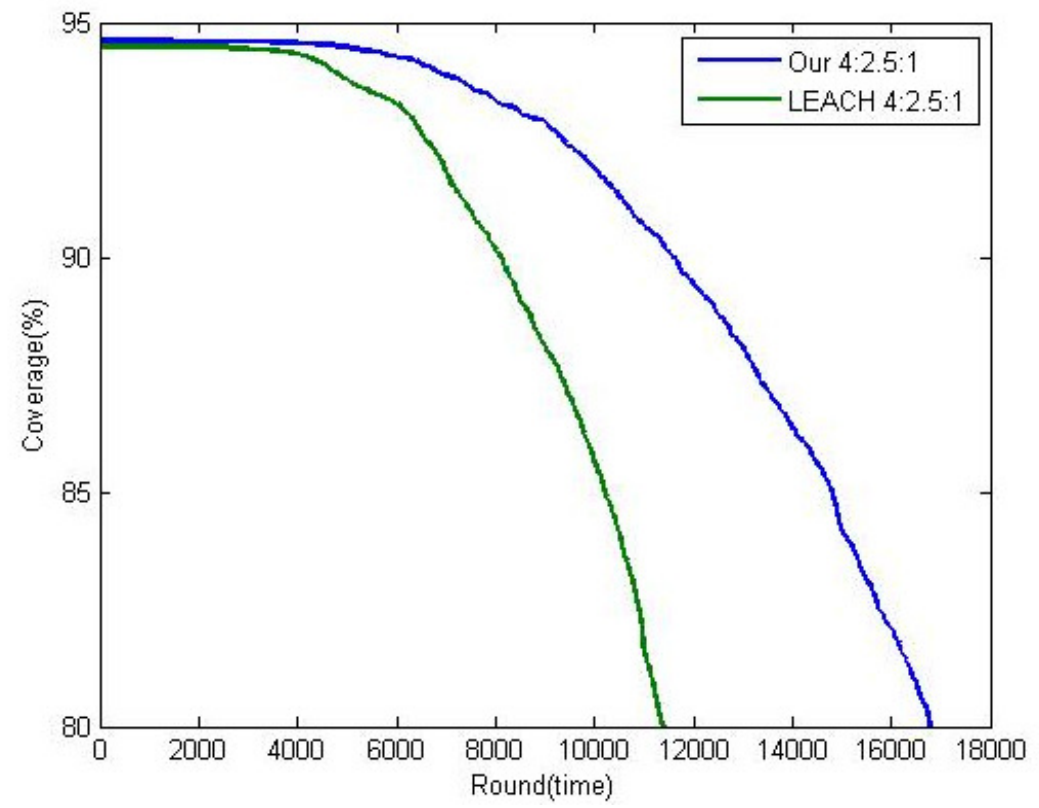

Figure 8. Comparing the results of both algorithms after density adjustment

Table 3. Initial coverage ratio and lifetime by adjusting node density in both algorithms

\begin{tabular}{|c|c|c|c|c|}
\hline \multirow{3}{*}{ Algorithm } & \multicolumn{2}{|c|}{ Initial coverage ratio } & \multicolumn{2}{c|}{$\begin{array}{c}\text { Rounds when coverage ratio } \\
\text { dropped below 80\% }\end{array}$} \\
\cline { 2 - 5 } & \multicolumn{2}{|c|}{ Density adjusted } & \multicolumn{2}{c|}{ Density adjusted } \\
\cline { 2 - 5 } & No & Yes & No & Yes \\
\hline LEECH & $99 \%$ & $95 \%$ & 5002 & 11379 \\
\hline proposed algorithm & $99 \%$ & $95 \%$ & 8342 & 16789 \\
\hline
\end{tabular}

\section{Conclusions}

How to deploy sensor nodes to cooperate with efficient routing algorithms for extending lifetime is an important topic in the research of WSN. For large-area applications, most routing algorithms adopt indirect transmission mode to solve the problem of high energy consumption due to long-distance transmission, but it may also cause the sensor nodes near the base station to exhaust electricity quickly. This study proposed a cluster allocation and routing algorithm based on node density to remain a high coverage ratio and thus extend the lifetime of WSN. The simulation results showed that the lifetime (in rounds) for the proposed algorithm was $66 \%$ higher than that of LEACH.

In addition, this study proposed a deployment method to adjust node density without increasing the total number of sensor nodes. The objective was to increase the sensor nodes near the base station to compensate for the high energy consumption due to forwarding data for other nodes. The simulation results showed that it was very efficient in increasing the lifetime of WSN. For the proposed algorithm and LEACH, their lifetimes were increased by more than $100 \%$ if the density proportion of 4:2.5:1 was used for node deployment. Therefore, the cluster allocation 
and routing algorithm as well as the density deployment method proposed in this study are very efficient in extending the lifetime of WSN.

\section{ACKNOWLEDGEMENTS}

The authors would like to thank for the financial support of the National Science Council (NSC) in Taiwan under the contract numbers NSC 100-2511-S-134-003.

\section{REFERENCES}

[1] I. F. Akyildiz, W. Su, Y. Sankarasubramaniam, and E. Cayirci, Wireless Sensor Networks: a Survey, Computer Networks, vol. 38, no. 4, pp. 393-422, 2002.

[2] W. R. Heinzelman, A. Chandrakasan, and H. Balakrishnan, Energy-efficient communication protocol for wireless microsensor networks, Proceedings of the 33rd Hawaii International Conference on System Sciences, vol. 8, pp. 8020, Jan. 4-7, 2000.

[3] K. Sohrabi, J. Gao, V. Ailawadhi, and G. J. Pottie, Protocols for self-organization of a wireless sensor network, IEEE Personal Communications, vol. 7, no. 5, pp. 16-27, 2000.

[4] Harish. H. Kenchannavar, Sushma. S. Kudtarkar, U. P. Kulkarni, Energy Efficient Data Processing in Visual Sensor Network, International Journal of Computer Science and Information Technology, vol.2, no.5, pp. 151-160, 2010.

[5] Laiali Almazaydeh, Eman Abdelfattah, Manal Al-Bzoor, and Amer Al-Rahayfeh, Performance Evaluation of Routing Protocols in Wireless Sensor Networks, International Journal of Computer Science \& Information Technology, vol.2, no.2, pp. 64-73, 2010.

[6] M. J. Handy, M. Haase and D. Timmermann, LEACH-C : Low energy adaptive clustering hierarchy with deterministic cluster-head selection, 4th IEEE International Conference on Mobile and Wireless Communication Network, pp. 368-372, Sep. 2002.

[7] S. Lindsey and C. S. Raghavendra, PEGASIS: Power efficient gathering in sensor information systems, Proceedings of the IEEE Aerospace Conference, vol. 3, pp. 1125-1130, 2002.

[8] A. Manjeshwar and D. P. Agrawal, TEEN: a routing protocol for enhanced efficiency in wireless sensor networks, Parallel and Distributed Processing Symposium, pp. 2009-2015, Apr. 2001.

[9] A. Manjeshwar and D. P. Agrawal, APTEEN: a hybrid protocol for efficient routing and comprehensive information retrieval in wireless sensor networks, Proceedings of the International Parallel and Distributed Processing Symposium, pp. 195-202, 2002.

[10] S. Meguerdichian, F. Koushanfar, M. Potkonjak and M. Srivastava, Coverage problems in wireless ad-hoc sensor networks, Proceedings of IEEE INFOCOM vol. 3, pp. 1380-1387, Apr. 2001.

[11] R. Ghrist and A. Muhammad, Coverage and hole-detection in sensor networks via homology, Proceedings of the 4th International Symposium on Information Processing in Sensor Networks, pp. 254-260, Apr. 24-27, 2005.

[12] Wernhuar Tarng, Kuo-Liang Ou, Kun-Jie Huang, Li-Zhong Deng, Hao-Wei Lin, Chang Wu Yu, Kuen-Rong Hsieh and Mingteh Chen, Applying Cluster Merging and Dynamic Routing Mechanisms to Extend the Lifetime of Wireless Sensor Networks, International Journal of Communication Networks and Information Security, vol. 3, no. 1, pp. 8-16, 2011.

[13] X. Tang and J. Xu, Extending network lifetime for precision-constrained data aggregation in wireless sensor networks, Proceedings of IEEE INFOCOM, Apr. 2006.

[14] J. M. S. N., L. B. Ruiz and A. F. Loureiro, Manna: A management architecture for wireless sensor networks, IEEE Communication Magazine, vol. 41, pp. 116-125, 2003.

[15] V. Raghunathan, C. Schurgers, S. Park, and M. B. Srivastava, Energy-aware wireless micro sensor networks, IEEE Signal Processing Magazine, vol. 19, no. 2, pp. 40-50, 2002.

[16] W. B. Heinzelman, P. Chandrakasan, and H. Balakrishnan, An application-specific protocol architecture for wireless micro sensor networks, IEEE Transactions on Wireless Communications, vol. 1, no. 4, pp. 660-670, 2002. 